\title{
Whole Genome Sequence based Capsular Typing and Antimicrobial Resistance Prediction of Group B Streptococcal Isolates from Colonized Pregnant Women in Nigeria
}

\author{
Mienye Bob-Manuel \\ Rivers State University Teaching Hospital \\ Lesley McGee ( $\square$ Imcgee@cdc.gov ) \\ Centers for Disease Control and Prevention \\ Jeremiah Igunma \\ University of Benin Teaching Hospital \\ Mary Alex-Wele \\ University of Port Harcourt Teaching Hospital \\ Orikomaba Obunge \\ University of Port Harcourt Teaching Hospital \\ Kennedy Wariso \\ University of Port Harcourt Teaching Hospital
}

\section{Research Article}

Keywords: Whole genome sequencing, Group B Streptococcus, Nigeria, capsular typing, antimicrobial resistance

Posted Date: April 28th, 2021

DOl: https://doi.org/10.21203/rs.3.rs-395069/v1

License: (c) (i) This work is licensed under a Creative Commons Attribution 4.0 International License. Read Full License 


\section{Abstract}

Background: Streptococcus agalactiae (Group B Streptococcus, GBS) is one of the major bacterial pathogens responsible for neonatal sepsis. Whole genome sequencing has, in recent years, emerged as a reliable tool for capsular typing and antimicrobial resistance prediction. This study characterized vaginal and rectal isolates of Group B Streptococcus obtained from pregnant women in Port Harcourt, Nigeria using a whole-genome sequence-based approach.

Results: Capsular types Ia, Ib, II, III, IV and V were detected among the 43 isolates sequenced. Twelve sequence types (STs) were identified, with ST19 $(n=9,27.3 \%)$ and ST486 $(n=5,15.2 \%)$ the most frequent among non-duplicated isolates. Of the alpha-like proteins (alp) identified, Alp1 was the most prevalent in 11 (33.3\%) isolates. Macrolide and lincosamide resistance determinants were present in 15 (45.5\%) isolates; ermB was detected in $1(3 \%)$ and ermTR in $7(21.2 \%)$ isolates. The Inu gene, was detected in 6 $(18.2 \%)$ and mef was identified in $3(9.1 \%)$ isolates. Resistance of GBS to erythromycin and clindamycin was found to be $30.3 \%$ and $24.2 \%$, respectively. All isolates were resistant to tetracycline with only the tet $M$ gene identified. Fluoroquinolone-resistance conferring substitutions in $g y r A+p a r C$ were detected in $9(27.3 \%)$ isolates and chloramphenicol resistance was predicted in 11 (33.3\%).

Conclusion: The data available from the whole genome sequencing of these isolates offers a small but insightful description of common serotypes and resistance features within colonizing GBS in Nigeria.

\section{Introduction}

Streptococcus agalactiae, also known as Group B Streptococcus (GBS) is increasingly being recognized in Africa as one of the major pathogens responsible for neonatal sepsis ${ }^{1-3}$. Vertical transmission from a rectovaginally colonized mother is the main predisposing factor for the development of early onset neonatal GBS sepsis ${ }^{4,5}$. Some countries have policies regarding intrapartum antibiotic prophylaxis (IAP) to prevent early-onset disease (EOD) with resulting benefits ${ }^{6,7}$. However, many low and middle income countries are yet to develop or implement such policies ${ }^{8}$. Whilst vaccine development is still underway 9,10 , IAP remains one of the most effective tools for the prevention of EOD ${ }^{7,11}$. The use of IAP may inadvertently result in the increase of antimicrobial resistance $(A M R)^{8}$. For this reason, determination of antimicrobial resistance patterns is important, especially settings such as in Nigeria where there is high incidence of indiscriminate antibiotic use ${ }^{12}$. Whole genome sequencing (WGS) has emerged as a reliable and cost-effective method for capsular typing and for accurate predictions of minimum inhibitory concentrations for antimicrobials in $\mathrm{GBS}^{13,14,15}$.

This study analyses whole genome sequenced vaginal and rectal isolates of Group B Streptococcus obtained from previously described study in pregnant women in Port Harcourt, Nigeria ${ }^{16}$. Data from this study will add to existing evidence to guide vaccine efforts as well as contribute to local recommendations for intrapartum antibiotic prophylaxis. 


\section{Materials And Methods}

\section{Bacterial Isolates}

A total of 46 isolates, obtained from separate vaginal and rectal samples of 185 pregnant women in their third trimester, collected retrospectively between March and July 2018 were used in this study. Samples were collected from women receiving antenatal care at the University of Port Harcourt Teaching Hospital in Port Harcourt Nigeria. Ethical approval was received from the hospital's ethics board and each participant signed a consent form prior to enrolment. Details of the study design and sample collection are described previously by Bob-Manuel et $\mathrm{al}^{16}$. Briefly, the rectovaginal samples were incubated overnight in LIM RambaQUICK ${ }^{\mathrm{TM}}$ and subcultured onto CHROMagar ${ }^{\mathrm{TM}}$ StrepB (CHROMagar, France). Streptococcus agalactiae were presumptively identified as small, mauve-coloured colonies on $\mathrm{CHROMagar}$ StrepB. Isolates were stored in tryptic soy broth containing $5 \%$ glycerol at $-70^{\circ} \mathrm{C}$. The isolates were shipped on Columbia agar with chocolated horse blood (Oxoid, UK) to the Streptococcus Laboratory, Centers for Disease Control and Prevention (CDC), for whole genome sequencing.

\section{Whole genome sequencing and strain feature prediction}

At CDC, GBS isolates were cultured on Trypticase soy agar supplemented with $5 \%$ sheep blood prior to DNA extraction from single colonies as previously described ${ }^{13}$. Following shearing of samples using a Covaris M220 ultrasonicator (Covaris, Inc, MA, USA) genomic libraries were constructed using sparQ DNA Library Kit (Quantabio, MA USA) ${ }^{13,14}$. Short read sequences were generated on Illumina Miseq platform with MiSeq v2 500 cycle kit (Illumina Inc) and assembled de novo using VelvetOptimiser. Serotypes, multilocus sequence typing (MLST), antibiotic resistance determinants, and predicted minimum inhibitory concentrations (MICs) were determined using a previously validated GBS bioinformatics pipeline $e^{13}$. Isolates flagged for phenotypic testing (those with Inu gene and ermTR $+\ln u$ ) were tested by Etest (Biomerieux USA), according to manufacturer's instructions. Additional strain features, including surface protein genes encoding the hypervirulent GBS adhesin ( $h v g a)$, serine-rich repeat ( $s r$ ) proteins, alpha protein family (alpha, Rib, Alp2/3, Alp1), and pilus proteins predictive of pilus islands (designated here as $\mathrm{PI} 1, \mathrm{PI} 2 \mathrm{~A}$, and PI2B) were extracted from genomic data (https://github.com/BenJamesMetcalf) ${ }^{14}$. Isolate identifiers, pipeline features, assembly metrics and NCBI genome accession numbers are listed in Supplementary Table 1.

\section{Results}

The proportion of vaginal/rectal samples positive for GBS was 16.8\% (31/185) (sTable 1); 12 women had both vaginal and rectal swabs positive for GBS with 10 having identical serotypes and therefore counted only once in further analyses. Whole genome sequencing revealed 3 of the isolates to be non-GBS. Six of ten known capsular serotypes (la, Ib, II, III, IV and V) were detected within this sampling, with type $\mathrm{V}$ the most frequent ( $n=11 / 33,33.3 \%$ )(Table 1). Multilocus sequence typing (MLST) identified a total of 12 STs among the 33 strains with ST19 $(n=9,27.3 \%)$ and ST486 $(n=5,15.2 \%)$ most frequently occurring. 
Eight (88.9\%) of the ST19 sequence strains were serotype V isolates while serotype III accounted for 1 (11.1\%) isolate. Only 1 serotype III isolate (3\%) had the highly virulent ST17 clonal type.

Table 1

Distribution of sequence types (ST) by serotype for 33 non-duplicate GBS isolates

\begin{tabular}{|c|c|c|c|c|c|c|}
\hline \multirow[t]{2}{*}{ ST } & \multicolumn{6}{|c|}{ Serotypes (\%) } \\
\hline & la & lb & II & III & IV & V \\
\hline 1 & - & - & - & - & - & $1(9.1)$ \\
\hline 2 & - & - & - & - & $2(100.0)$ & - \\
\hline 8 & - & $1(100)$ & - & - & - & - \\
\hline 17 & - & - & - & 1 (14.3) & - & - \\
\hline 19 & - & - & - & $1(14.3)$ & - & $8(72.7)$ \\
\hline 23 & $1(16.7)$ & - & - & - & - & - \\
\hline 24 & $4(66.7))$ & - & - & - & - & - \\
\hline 26 & - & - & - & - & - & $1(9.1)$ \\
\hline 28 & - & - & 2 (33.3) & - & - & $1(9.1)$ \\
\hline 182 & - & - & - & $3(42.9)$ & - & - \\
\hline 486 & $1(16.7)$ & - & $4(66.7)$ & - & - & - \\
\hline 1336 & - & - & - & 2 (28.6) & - & - \\
\hline Total & 6 (18.2) & $1(3.0)$ & $6(18.2)$ & $7(21.2)$ & $2(6.1)$ & 11 (33.3) \\
\hline
\end{tabular}

The most predominant alpha-protein-like (alp) family present was Alp1 in 11 (33.3\%) isolates, followed by Rib with 9 (27.3\%), Alp2/3 with 6 (18.23\%) isolates and 5 (15.2\%) isolates were Alpha. Rib was detected in capsular types II, III and V; alp2/3 was detected in Ia, II and V; alp 1 in Ia, IV and V while the Alpha protein was detected in type la and $\mathrm{lb}$ alone. Two isolates were negative for any of the alpha-protein-like family target predictions.

Serine-rich repeat proteins SSR1 and SSR2 were predicted in $29(87.9 \%)$ and 1 (3\%) of the isolates, while $3(9.1 \%)$ were negative for both. Only one isolate demonstrated $h v g A$, a serotype III ST17 isolate as expected, the same isolate that was SSR2 positive. Isolates either had 1 or 2 pili; PI-1 + PI2A detected in 19 (57.6\%), PI1 + PI2B in 8 (24.2\%) and PI2A in 6 (18.1\%) isolates.

Table 2 shows the antibiotic susceptibility as predicted by WGS and detection of specific resistance determinants. Macrolide and clindamycin resistance determinants were present in 15 (45.5\%) of the 
isolates with resistance rates to erythromycin and clindamycin of $30.3 \%$ and $24.2 \%$, respectively. These determinants were present only in serotypes III, IV and V. The $\mathrm{MLS}_{\mathrm{B}}$ genes which confer resistance to macrolides, lincosamides and streptogramin $\mathrm{B}, \mathrm{ermB}$ was detected in $1(3.0 \%)$ isolates and ermTR in 7 (21.2\%) isolates; lincosamide nucleotidyltransferase determinant, Inu, was present in $6(18.2 \%)$ and the determinant for macrolide efflux, mef was detected in $3(9.1 \%)$ isolates. The $5(15.2 \%)$ isolates with Inu alone and the one isolate with ermTR + Inu were susceptible to erythromycin + clindamycin and synercid, respectively, when tested phenotypically by Etest. Isolates with $m e f / m s r D$ alone $(2 ; 6.1 \%)$ were predicted as resistant to erythromycin but sensitive to clindamycin.

Table 2

Whole Genome Sequencing predicted antibiotic susceptibility for 33 non-duplicate GBS isolates

\begin{tabular}{|lll|}
\hline Antibiotic & Susceptible & Non-Susceptible \\
\hline Ampicillin & $33(100)$ & - \\
\hline Ceftriaxone & $33(100)$ & - \\
\hline Chloramphenicol & $25(75.6)$ & $8(24.2)$ \\
\hline Clindamycin & $25(75.6)$ & $8(24.2)$ \\
\hline Ceftaroline & $33(100)$ & - \\
\hline Daptomycin & $33(100)$ & - \\
\hline Erythromycin & $23(69.7)$ & $10(30.3)$ \\
\hline Levofloxacin & $24(72.7)$ & $9(27.3)$ \\
\hline Meropenem & $33(100)$ & - \\
\hline Linezolid & $33(100)$ & - \\
\hline Penicillin & $33(100)$ & - \\
\hline Synercid & $33(100)$ & - \\
\hline Cefotaxime & $33(100)$ & - \\
\hline Tetracycline & - & $33(100)$ \\
\hline Vancomycin & $33(100)$ & - \\
\hline * Quinupristin/Dalfopristin & \\
\hline
\end{tabular}

All isolates were resistant to tetracycline and the only gene detected conferring resistance was tetM. Fluoroquinolone-resistance conferring substitutions in both gyrA and parC were present in 9 (27.3\%) isolates conferring full resistance phenotype, 8 (88.9\%) of which were ST19 strains. Also, all of the isolates with fluoroquinolone resistance determinants were serotype $\mathrm{V}$. The aac6'-aph2, which encodes 
the bifunctional aminoglycoside-inactivating enzyme with 6'-acetyltransferase and 2"phosphotransferase activities, were detected in 11 (33.3\%) isolates and were exclusively in serotypes III, IV and V: 4 (36.4\%), 1 (9.1\%) and 6 (54.5\%), respectively. CAT genes, encoding chloramphenicol acetyltransferases, were detected in $8(24.2 \%)$ isolates, among serotypes III, IV and V.

\section{Discussion}

This study reports the molecular characterization of colonizing strains of Group B Streptococcus in the vagina and rectum of pregnant women. Serotype $V$ was the most frequent serotype in this population representing $33.3 \%$ of the population.

Sequence types ST19, 486, 182, 24 and 2 accounted for $69.7 \%$ of the total isolates. This diversity is different from other reports that have reported ST19, ST23 and ST1 more commonly ${ }^{17}$. The highly virulent ST17 responsible for most invasive disease, especially late onset disease in neonates ${ }^{18}$, was rare in this study (3\%) population which may suggest a better prognosis for neonates who may become colonized at birth in this environment. While ST182 was reported as a frequent clonal type by Medugu et al in another local study in Abuja, Nigeria ${ }^{2}$, ST486 has not previously been documented as a common sequence type among GBS isolates in Nigeria.

Alp1 (Epsilon) (33.3\%) was the most common of all the alpha-like proteins among the isolates in this study, similar to findings reported in Egypt $(27 \%)^{19}$. Other studies have documented Rib as the most frequently detected Alp gene in other geographic regions like China and Iran $(37.5 \%$ and $53 \%$ respectively) ${ }^{20,21}$. The serine-rich repeat protein Srr-1 was identified in almost all isolates, however only a single isolate had the Srr-2 known to be associated with greater binding affinity and higher morbidity 22 . The same isolate was positive for the hypervirulent GBS adhesin ( $\mathrm{HvgA})$, another surface protein demonstrated to increase adhesion and virulence particularly in the ST17 lineage ${ }^{23}$.

Similar to other studies from Nigeria ${ }^{2,24}$, all isolates were determined to be susceptible to beta-lactam antibiotics including 3rd generation cephalosporins. This is quite reassuring, suggesting that penicillin and ampicillin remain as viable options for intrapartum antibiotic prophylaxis in this region despite concerns for antimicrobial resistance. For women allergic to penicillin, guidelines recommend clindamycin as an alternative option for prophylaxis ${ }^{25}$. In this study however, clindamycin and erythromycin resistance were relatively high at $24.2 \%$ and $30.3 \%$, respectively. Data from an earlier study in Nigeria reported erythromycin and clindamycin resistance rates of $6.5 \% 24$ suggesting increased resistance rates within the country. However, all isolates were susceptible to vancomycin in this study, so patients with a high risk for penicillin anaphylaxis who are colonized with a clindamycin-resistant GBS strain, could receive vancomycin as the next best option ${ }^{25}$.

Although other antibiotic classes such as fluoroquinolones, chloramphenicol, and tetracycline are not recommended for intrapartum prophylaxis, determining their susceptibility is important for tracking of resistance at the pneumococcal population level and as useful options for other invasive group B 
streptococcal infections in other age groups. All isolates were predicted as tetracycline resistant which is consistent with several other reports documenting high levels of resistance to this antibiotic 24,26,27. Resistance to chloramphenicol and levofloxacin were also high at $25.6 \%$. While these antibiotics may not be recommended for neonatal sepsis, (for which we screen mothers for GBS to prevent), they are useful options for other invasive group B streptococcal infections in other age groups.

The only tetracycline gene present in this population was tetM. Of all the tetracycline resistance determinants, the tetM has the most spread geographically among $\mathrm{GBS}^{24,26,27}$. The presence of the double gyrA + parC mutants conferring full fluoroquinolone-resistance was high and were found predominantly in serotype V/ST-19 clone. The most frequently reported fluoroquinolone non-susceptible serotype/ST combinations are III/ST-19 and V/ST-19 28,29 supporting the data from this study. Occurrence of either of these clonal complexes is likely a reflection of the circulating clones in those geographical areas in which they are found.

This study has demonstrated some salient characteristics of the prevalent colonizing strains of GBS in Nigeria, thus, contributing to the body of knowledge concerning GBS, as well as providing AMR data if IAP is considered in the future.

\section{Declarations}

Ethics approval and consent to participate: Ethical approval for the study was obtained from the University of Port Harcourt Teaching Hospital Ethical Committee (UPTH/ADM/90/S. II/VOL.XI/369). Written informed consent was obtained from each participant prior to study enrolment. All methods were carried out in accordance with relevant guidelines and regulations.

Consent for publication: All authors contributed to study and manuscript development and consent to publication.

Availability of data and materials: Genome sequences has been uploaded to GenBank https://www.ncbi.nIm.nih.gov/sra/PRJNA632041. See sTable 1 for Biobank Accession numbers (SAMN18352391-SAMN18352411).

Competing interests: The authors declare that they have no competing interests.

Funding: Funding for whole genome sequencing was provided by CDC through Advanced Microbial Detection Project FY21-AMD-161.

Authors' contributions: Authors MB and LM conceived and designed the study. LM coordinated the whole genome sequencing and analyses. MB wrote most of the manuscript with major review and editing by LM. JI and MA helped with the data analysis. $\mathrm{OO}$ and $\mathrm{KW}$ contributed to critically reviewing the manuscript. All authors read and approved the final manuscript. 
Acknowledgements: Sincere thanks to Bernard Beall, Chief Streptococcus Laboratory, Centers for Disease control and Prevention and his entire team for their willingness to assist and making this strain characterization study possible.

The findings and conclusions in this report are those of the authors and do not necessarily represent the official position of the Centers for Disease Control and Prevention.

\section{References}

1. Yadeta TA, Worku A, Egata G, Seyoum B, Marami D, Berhane Y. Vertical transmission of group B streptococcus and associated factors among pregnant women: A cross-sectional study, Eastern Ethiopia. Infect Drug Resist. 2018; 11: 397-404. DOI: 2147/IDR.S150029.Accessed 29 March 2018.

2. Medugu N, Iregbu KC, Parker RE, Plemmons J, Singh P, Audu LI, et al. Group B streptococcal colonization and transmission dynamics in pregnant women and their newborns in Nigeria: implications for prevention strategies. Clin Microbiol Infect. 2017; 23(9): 673. e9-673.e16. DOI: 1016/j.cmi.2017.02.029. Accessed 22 March 2018.

3. Sinha A, Russell LB, Tomczyk S, Verani JR, Schrag SJ, Berkley JA, et al. Disease burden of Group B streptococcus among infants in Sub-Saharan Africa. Pediatr Infect Dis J. 2016;35(9): 933-942. DOI: 1097/INF.0000000000001233. Accessed 29 March 2018.

4. Dangor Z, Lala SG, Cutland CL, Koen A, Jose L, Nakwa F, et al. Burden of invasive group B Streptococcus disease and early neurological sequelae in South African infants. PLoS One. 2015; 10(4). DOI: 1371/journal.pone.0123014. Accessed 29 March 2018.

5. Hansen SM, Uldbjerg N, Kilian M, Sørensen UBS. Dynamics of Streptococcus agalactiae Colonization in Women during and after Pregnancy and in Their Infants. J Clin Microbiol. 2004; 42(1): 83-89. DOI: 1128/jcm.42.1.83-89.2004. Accessed 22 March 2018.

6. Daley AJ, Isaacs D. Ten-Year Study on the Effect of Intrapartum Antibiotic Prophylaxis on Early Onset Group B Streptococcal and Escherichia coli Neonatal Sepsis in Australasia. Pediatr Infect Dis J. 2004;23(7): 630-63 DOI: 10.1097/01.inf.0000128782.20060.79. Accessed 01 April 2018.

7. Schrag SJ, Verani JR. Intrapartum antibiotic prophylaxis for the prevention of perinatal group $B$ streptococcal disease: Experience in the United States and implications for a potential group B streptococcal vaccine. Vaccine. 2013;31: D20-2 DOI: 10.1016/j.vaccine.2012.11.056. Accessed 2 September 2018.

8. Le Doare K, O'Driscoll M, Turner K, Seedat F, Russell NJ, Seale AC, et al. Intrapartum Antibiotic Chemoprophylaxis Policies for the Prevention of Group B Streptococcal Disease Worldwide: Systematic Review. Clin Infect Dis. 2017; 65(Suppl 2): S143-151. DOI: 1093/cid/cix654. Accessed 10 March 2020.

9. Kobayashi M, Schrag SJ, Alderson MR, Madhi SA, Baker CJ, Sobanjo-ter Meulen A, et al. WHO consultation on group B Streptococcus vaccine development: Report from a meeting held on 27-28 
April 2016. Vaccine. 2016; 37 (50): 7307-7314. DOI: 1016/j.vaccine.2016.12.029. Accessed 27 February 2018.

10. Lin SM, Zhi Y, Ahn KB, Lim S, Seo HS. Status of group B streptococcal vaccine development. Clin Exp Vaccine Res. 2018; 7(1): 76. DOI: 7774/cevr.2018.7.1.76. Accessed 10 March 2020.

11. Nishihara Y, Dangor Z, French N, Madhi S, Heyderman R. Challenges in reducing group $B$ Streptococcus disease in African settings. Arch Dis Child. 2017; 102(1): 72-77. DOI: 1136/archdischild-2016-311419. Accessed 10 March 2020.

12. Egwuenu A, Obasanya J, Okeke I, Aboderin O, Olayinka A, Kwange D, et al. Antimicrobial use and resistance in Nigeria: situation analysis and recommendations, 2017. Pan Afr Med J. 2018; 8(8):2. DOI: 11604/pamj.cp.2018.8.2.701. Accessed 2 June 2020.

13. Metcalf BJ, Chochua S, Gertz RE, Hawkins PA, Ricaldi J, Li Z, et al. Short-read whole genome sequencing for determination of antimicrobial resistance mechanisms and capsular serotypes of current invasive Streptococcus agalactiae recovered in the USA. Clin Microbiol Infect. 2017;23(8): 574.e7-574.e14. DOI: 1016/j.cmi.2017.02.021. Accessed 23 January 2019.

14. McGee L, Chochua S, Li Z, Mathis S, Rivers J, Metcalf B, et al. Multistate, population-based distributions of candidate vaccine targets, clonal complexes, and resistance features of invasive Group B Streptococci within the US: 2015-2017. Clin Infect Dis. 2020 Feb 15:ciaa151.

DOI: 10.1093/cid/ciaa151. Accessed 6 December 2020.

15. Zankari E, Hasman H, Cosentino S, Vestergaard M, Rasmussen S, Lund O, et al. Identification of acquired antimicrobial resistance genes. J Antimicrob Chemother. 2012; 67(11): 2640-2644. DOI: 1093/jac/dks261. Accessed 3 March 2020.

16. Bob-Manuel M, Lawson SD, Kasso T, Oboro IL, Obunge OK, Wariso KT. Epidemiology and Capsular Typing of Group B Streptococcus in Pregnant Women in Port Harcourt, Nigeria. Annals Microbiol Infect Dis. 2021; 4(1): -16.

17. Manning SD, Lewis MA, Springman AC, Lehotzky E, Whittam TS, Davies HD. Genotypic diversity and serotype distribution of group B streptococcus isolated from women before and after delivery. Clin Infect Dis. 2008; 46(12): 1829-18 DOI: 10.1086/588296. Accessed 30 September 2020.

18. Jones N, Oliver KA, Barry J, Harding RM, Bisharat N, Spratt BG, et al. Enhanced invasiveness of bovine-derived neonatal sequence type 17 group $B$ Streptococcus is independent of capsular serotype. Clin Infect Dis. 2006; 42(7):915-924. DOI: 1086/500324. Accessed 28 October 2020.

19. Shabayek S, Abdalla S. Abouzeid A MH. Serotype and surface protein gene distribution of colonizing group B streptococcus in women in Egypt. Infect. 2014; 142:208-210.

DOI: 10.1017/S0950268813000848. Accessed 2 November 2020.

20. Lu B, Wang D, Zhou H, Zhu F, Li D, Zhang S, et al. Distribution of pilus islands and alpha-like protein genes of group B Streptococcus colonized in pregnant women in Beijing, China. Eur J Clin Microbiol Infect Dis. 2015; 34(6):1173-1179. DOI: 1007/s10096-015-2342-9. Accessed 20 March 2018.

21. Sadeh M, Firouzi R, Derakhshandeh A, Bagher Khalili M, Kong F, Kudinha T. Molecular Characterization of Streptococcus agalactiae Isolates From Pregnant and Non-Pregnant Women at 
Yazd University Hospital, Iran. Jundishapur J Microbiol. 2016;9(2):e30412. DOI: 5812/jjm.30412. Accessed 2 February 2018.

22. Armistead B, Oler E, Adams Waldorf K, Rajagopal L. The Double Life of Group B Streptococcus: Asymptomatic Colonizer and Potent Pathogen. J Mol Biol. 2019; 431(16): 2914-2931. DOI: 1016/j.jmb.2019.01.035. Accessed 2 November 2020.

23. Tazi A, Disson O, Bellais S, Bouaboud A, Dmytruk N, Dramsi S, Mistou MY, et al. The surface protein HvgA mediates group B streptococcus hypervirulence and meningeal tropism in neonates. J Exp Med. 2010; 207(11): 2313-23 DOI: 10.1084/jem.20092594. Accessed 30 September 2020.

24. Elikwu CJ, Oduyebo O, König B. Antibiotic susceptibility profiles of group B streptococci (GBS) isolates from pregnant mothers in a tertiary institution in Nigeria. International Journal of Infectious Diseases. 2014;21:348. DOI: 10.4103/2468-185250. Accessed 25 February 2018.

25. Prevention of Group B Streptococcal Early-Onset Disease in Newborns: ACOG Committee Opinion, Number 797. Obstet Gynecol. 2020; 135(2):e51-e72. DOI: 1097/AOG.0000000000003669. Accessed 2 November 2020.

26. Bergal A, Loucif L, Benouareth DE, Bentorki AA, Abat C, Rolain JM. Molecular epidemiology and distribution of serotypes, genotypes, and antibiotic resistance genes of Streptococcus agalactiae clinical isolates from Guelma, Algeria and Marseille, France. Eur J Clin Microbiol Infect Dis. 2015; 34(12): 2339-2348. DOI: 1007/s10096-015-2487-6. Accessed 2 September 2020.

27. Dutra VG, Alves VMN, Olendzki AN, Dias CAG, de Bastos AFA, Santos GO, et al. Streptococcus agalactiae in Brazil: Serotype distribution, virulence determinants and antimicrobial susceptibility. BMC Infect Dis. 2014; 14(1): 1-9. DOI: 1186/1471-2334-14-323. Accessed 2 November 2020.

28. Hays C, Louis M, Plainvert C, Dmytruk N, Touak G, Trieu-Cuot P, et al. Changing Epidemiology of Group B Streptococcus Susceptibility to Fluoroquinolones and Aminoglycosides in France. Antimicrob Agents Chemother. 2016; 60(12): 7424-7430. DOI: 1128/AAC.01374-16. Accessed 2 November 2020.

29. Wang P, Tong JJ, Ma XH, Song FL, Fan L, Guo CM, et al. Serotypes, antibiotic susceptibilities, and multi-locus sequence type profiles of Streptococcus agalactiae isolates circulating in Beijing, China. PLoS One. 2015; 10(3): 1-13. DOI: 1371/journal.pone.0120035. Accessed 28 March 2020.

\section{Supplementary Files}

This is a list of supplementary files associated with this preprint. Click to download.

- SupplementalTable1.xlsx 